\title{
INVESTIGATION OF SELF-HEALING BY USING ETHYL CELLULOSE ENCAPSULATED BACTERIUM IN CEMENTITIOUS MATERIALS
}

\author{
BING LIU ${ }^{1,2^{*}}$, XU DENG $^{3}$, JINLONG ZHANG ${ }^{3}$, NINGXU HAN ${ }^{1}$ AND FENG XING ${ }^{1,2}$ \\ ${ }^{1}$ College of Civil Engineering, Guangdong Provincial Key Laboratory of Durability for Marine Civil \\ Engineering, The Key Laboratory on Durability of Civil Engineering in Shenzhen, Shenzhen University, \\ Shenzhen 518060, PR China \\ e-mail: liubing0708@szu.edu.cn; nxhan@szu.edu.cn; xingf@szu.edu.cn \\ ${ }^{2}$ Shenzhen Institute of Information Technology \\ Shenzhen, 518172, P.R.China \\ ${ }^{3}$ College of Life Science, Shenzhen Key Laboratory of Marine Bioresource and Eco-environmental Science, \\ Shenzhen University, Shenzhen 518060, PR China \\ e-mail: dengxu@szu.edu.cn; greenwoodzjl@163.com
}

Key words: Calcium carbonate precipitation, Crack, Microbial microcapsules, Self-healing concrete, Durability

\begin{abstract}
A new approach to microcapsule based microbial self-healing system is presented that aims to heal the crack in cementitious materials. In this work, ethyl cellulose (EC) was designed to fabricate microcapsule as a protection strategy to encapsulate an alkaliphilic spore-forming bacterium. The technical feasibility of encapsulated spores and the influence factors were studied by calcium precipitation activity (CPA) of the bacterium. The CPA of broken/unbroken microcapsules was evaluated. The micro-morphology of the precipitation produced by the bacterium was investigated through Environmental Scanning Electron Microscopy (ESEM), X-ray Diffraction (XRD) and X-ray energy dispersive spectroscopy (EDS). X-ray Computed Tomography (XCT) was applied to trace the crack development and self-healing behavior of encapsulated mineralization bacterium in cement paste specimens in three dimensions.

The experimental results showed that compared with unbroken microcapsules, higher CPA was achieved by breaking the microcapsule to release the bacterium, suggesting good protection for the encapsulated spores. Subsequent production of calcium carbonate confirmed by ESEM and EDS indicated activation of encapsulated mineralization bacterium. The XCT results showed that formation of crack successfully triggered the breakage of embedded microcapsules. Compared with the specimens without embedded bacterium, the healed crack area of specimens embedded with bacterial microcapsules was monitored, suggesting effective self healing of concrete crack can be achieved by introducing encapsulated mineralization microorganisms into concrete structures.
\end{abstract}

\section{INTRODUCTION}

Formation of cracks inside the concrete during service is a main concern in civil engineering, as cracks may lead to ingress of air, water and aggressive substance into the concrete, consequently causing the deterioration of internal structure. Microbially induced self healing of concrete crack has been proved to be a promising strategy. However, due to the fact that microorganisms must be imbedded in the form of spore and spores are subject to being crushed as the inner pore size of concrete tends to decrease during the curing time, how to protect the embedded 
spores from crushing and high $\mathrm{pH}$ during the long period of hydration process and how to trigger the healing process at the moment of crack appearance in the cementitious matrix are very important. For a microcapsule-based microbial self-healing system in the cemetitous material, a fundamental issue is to find a suitable microcapsule system, concerning both the material selection and manufature process. In this study, a novel selfhealing microcapsule was designed and fabricated, a $\mathrm{pH}$ sensitive material was used for the shell of microcapsule, and an alkaliphilic spore-forming bacterium was encapsulated as the healing agent inside the microcapsule.

\section{MATERIALS AND METHODS}

\subsection{Preparation of microcapsules}

Microcapsules were fabricated via extrusion- spheronisaion and spray drying method by using a $\mathrm{pH}$ sensitive material, Ethyl Cellulose (EC), and an alkaliphilic sporeforming bacterium, Bacillus pseudofirmus DSM8715.

First of all, the influence factors on DSM8715 spore production and activity, such as the optimum $\mathrm{pH}$ value to induce mineralization, the optimum germination agent, the optimum buffer, and four kinds of organic solvents (methylbenzene, ethanol, acetone and ethyl acetate), which were used for the fabrication of microcapsule, were studied by monitoring the calcium precipitation activity (CPA) of the bacterium.

In addition, microcrystalline cellulose (MCC) which was used as filling skeleton was mixed with spores to prepare the core material. The mixture was formed into pellets with a cold extrusion rounded pelletizing machine. In order to obtain a suitable solution for the shell of microcapsule, ethyl cellulose was dissolved by ethanol. Spray drying process was carried out to form the outer coating of the microcapsules.

\subsection{Characterization of the microcapsules}

The micro-morphology of microcapsule and the interface between microcapsule and cement paste was evaluated by Environmental Scanning Electron Microscopy (ESEM). The morphology of the precipitation produced by the bacterium was investigated through X-ray Diffraction (XRD), and X-ray energy dispersive spectroscopy (EDS). In addition, an alkaline $\mathrm{pH}$ condition was designed to characterize the releasing performance of the microcapsule under a simulated concrete environment. The release feature of capsule wall at different $\mathrm{pH}$ gradients is monitored by means of micro-plate spectrophotometer. Further, the CPA of broken/unbroken microcapsules was studied by high-throughput assay which based on the detection of free $\mathrm{Ca} 2+$ concentration via O-Cresolphthalein Complexone (OCPC) method.

\subsection{Healing process}

Bacterial spores were encapsulated and then microcapsules were introduced into cement paste specimens during mixture period. After 14 days hydration period, the specimens were subjected to a splitting test to make crack. The crack closure behavior of the specimens with/without bio-microcapsules was studied quantitatively by light microscopy. To visualize the self-healing process inside the specimens, the crack-healing process is monitored with the help of X-ray Computed Tomography (XCT). In addition, the healing mechanism is studied by means of ESEM and EDS. Furthermore, efficiency of self-healing performance was evaluated with healed crack width.

\section{RESULTS AND DISCUSSION}

Given the alkaline environment inside the concrete, the bacterium used in the study was basophilic Bacillus pseudofirmus DSM 8715. Our experiment results showed that DSM8715 had strong mineralization ability and grew fast at $30^{\circ} \mathrm{C}, \mathrm{pH} 9.5-11$. The influence factors on DSM8715 spore production were evaluated. The results showed that the optimum germination agent is inosine with the suitable concentration of $80 \mathrm{mmol} / \mathrm{L}$; the optimum buffer is $\mathrm{N}$-cyclohexyl-3-amino propylsulfonic acid (CAPS); the optimum $\mathrm{pH}$ for DSM8715 
to induce mineralization was 10 with spore mineralization activity being $86.8 \%$ (Figure 1 ). Compared with unbroken microcapsules, a higher CPA was achieved by breaking the microcapsule to release the bacterium, suggesting good protection for the encapsulated spores (Figure 2). Four organic solvents showed harmful influence on the spores, while ethanol was relatively less harmful.

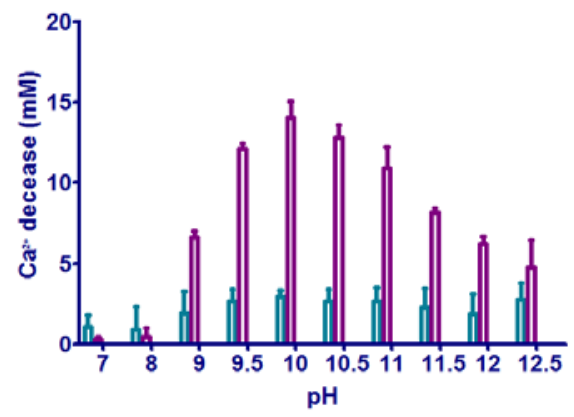

Figure 1: The influence of $\mathrm{pH}$ on the bacterial mineralization.

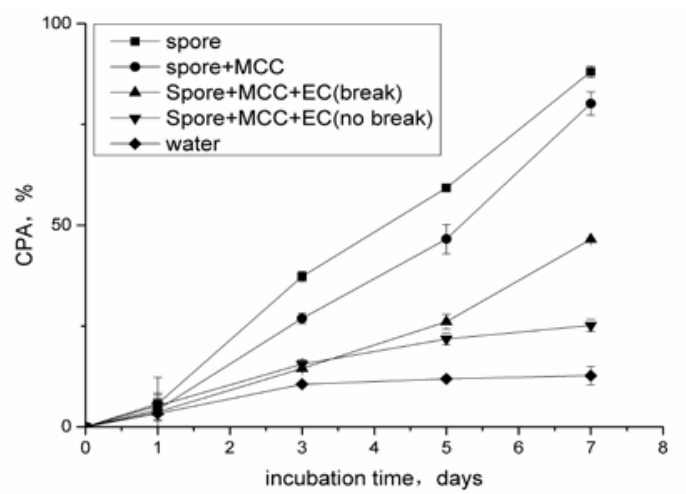

Figure 2: Comparison of CPA of bacteria in different forms.

In this study, self-healing behavior of encapsulated mineralization bacterium in cement paste specimens was investigated. The crack-introduced specimen was carefully studied to figure out the working mechanism of the trigger. Originally the microcapsule was able to maintain intact spherical structure. However, formation of crack resulted in breaking of microcapsule, subsequently leading to the release of encapsulated bacterial spores and initiation of self healing process. It was shown in Figure 3 and Figure 4 that microcapsules were fractured upon the formation of crack, indicating that the desired trigger system actually works. Subsequent production of calcium carbonate confirmed by EDS indicated activation of encapsulated mineralization bacterium (Figure 5).
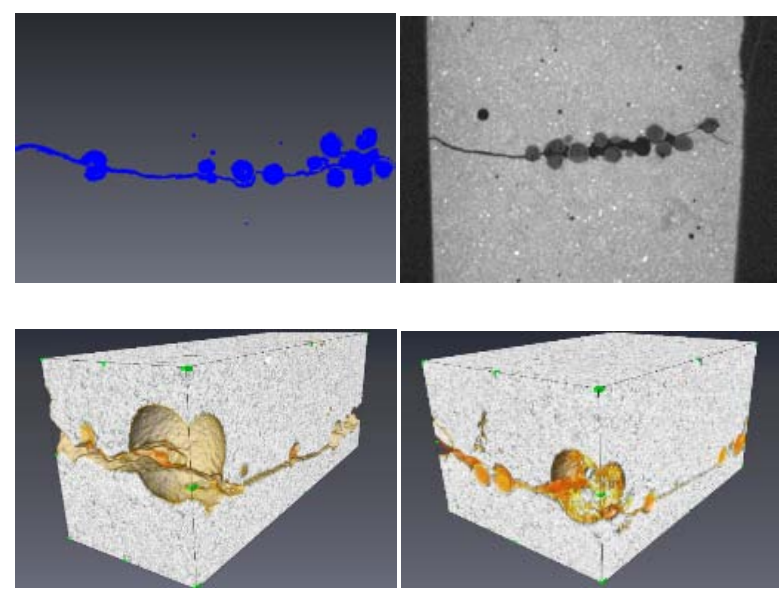

Figure 3: Breakages of microcapsules upon cracking and subsequent healing procedure were visually monitored by XCT in three dimensions.
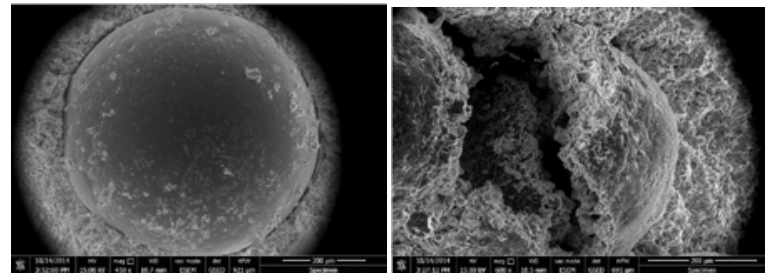

Figure 4: SEM image of microcapsule introduced in cement specimen.
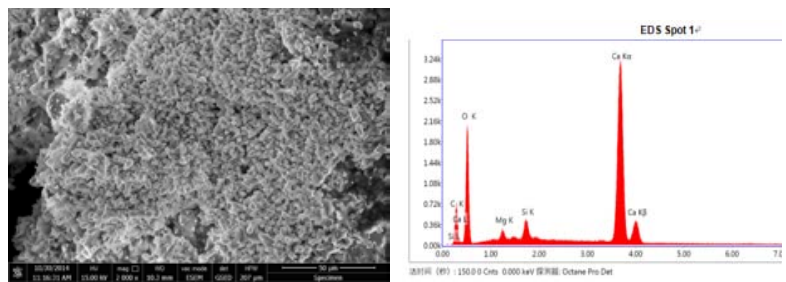

Figure 5: The crystal produced by the bacterium and its EDS result.

The crack-healing process, mechanism and healing effectiveness were studied to evaluate the feasibility of self-healing system. Compared with the specimens without embedded bacterium, the healed crack width of specimens embedded with bacterial microcapsules was higher and faster (Figure 6), suggesting effective self healing of concrete crack can be achieved by introducing encapsulated mineralization microorganisms 
into concrete structures.

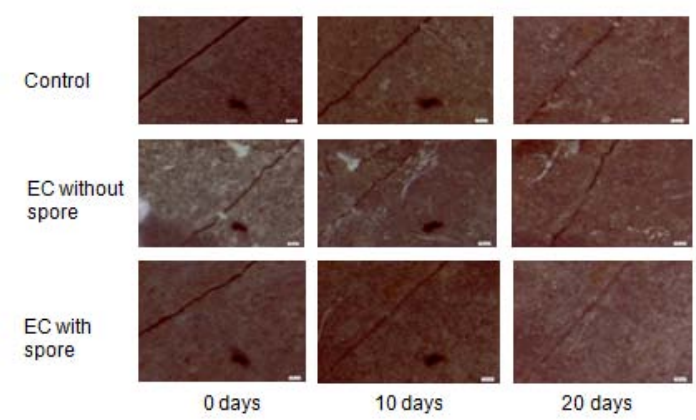

Figure 6: Breakage of crack and subsequent healing procedure was visually monitored by light microscope.

\section{CONCLUSION}

In this study, a microcapsule-based microbial self-healing system with Ethyl Cellulose and an alkaliphilic spore-forming bacterium was designed and fabricated. The physical properties and morphology of spore and microcapsules were evaluated for selfhealing system. The healing concept of biomicrocapsules based self-healing system was demonstrated to be feasible. More specifically, the physical trigger system was proved to be viable. In conclusion, all above discussed properties demonstrated that it is feasible to employ this microcapsule strategy to provide protection for mineralization bacteria in the self-healing process of concrete cracks.

\section{ACKNOWLEDGEMENTS}

The authors would like to acknowledge financial support provided by National Natural Science Foundation of China (No.51120185002, 51508338), Shenzhen R\&D Fund (JCYJ20140418091413530) and China Postdoctoral Science Foundation (2014M562209).

\section{REFERENCES}

[1] H. M. Jonkers, A. Thijssen, G. Muyzer, O. Copuroglu, E. Schlangen. 2010. Application of bacteria as self-healing agent for the development of sustainable concrete. Ecological engineering 36:230235.

[2] W. Virginie, H. M. Jonkers. 2011. Quantification of crack-healing in novel bacteria-based self-healing concrete.
Cement and Concrete Composites 33:763770.

[3] K. Van Tittelboom, N. De Belie. 2013. Self-healing in cementitious materials - A review. Materials 6: 2192-2217.

[4] J. Y. Wang, H. Soens, W. Verstraete, N. De Belie. 2014. Self-healing concrete by use of microencapsulated bacterial spores. Cement and Concrete Research 56 : 139152.

[5] J. Y. Wang, D. Snoeck, S. Van Vlierberghe, W. Verstraete, N. De Belie. 2014. Application of hydrogel encapsulated carbonate precipitating bacteria for approaching a realistic self-healing in concrete. Construction and Building Materials 68:110-119. 